\title{
Linearly equivalent topologies and locally quasi-unmixed rings
}

\author{
Adeleh Azari (D), Simin Mollamahmoudi (D), Reza Naghipour*(D) \\ Department of Mathematics, University of Tabriz, P.O. Pox: 51666-16471, Tabriz, Iran.
}

\begin{abstract}
Let $\bar{I}$ denote the integral closure of an ideal in a Noetherian ring $R$. The main result of this paper asserts that $R$ is locally quasi-unmixed if and only if, the topologies defined by $\overline{I^{n}}$ and $I^{\langle n\rangle}, n \geq 1$, are equivalent. In addition, some results about the behavior of linearly equivalent topologies of ideals under various ring homomorphisms are included.
\end{abstract}

Mathematics Subject Classification (2010). 13B22, 13E05

Keywords. associated primes, linearly equivalent topologies, integral closure, locally quasi-unmixed ring, Rees ring

\section{Introduction}

Let $R$ denote a commutative Noetherian ring, and $I$ an ideal of $R$. The interesting concept of quintasymptotic prime ideals of $I$ was introduced by McAdam [3]. A prime ideal $\mathfrak{p}$ of $R$ is called a quintasymptotic prime ideal of $I$ if there exists $z \in \operatorname{mAss}_{R_{\mathfrak{p}}^{*}} R_{\mathfrak{p}}^{*}$ such that $\operatorname{Rad}\left(I R_{\mathfrak{p}}^{*}+z\right)=\mathfrak{p} R_{\mathfrak{p}}^{*}$. The set of quintasymptotic prime ideals of $I$ is denoted by $\bar{Q}^{*}(I)$, and it is a finite set. Also, in [10], L. J. Ratliff, Jr., introduced the set of associated primes $\bar{A}^{*}(I):=\operatorname{Ass}_{R} R / \overline{I^{n}}$ for large $n$, called the presistent prime ideals of $I$, and he showed that this finite set has some nice properties in the theory of asymptotic prime divisors; here for any ideal $J$ of $R, \bar{J}$ denotes the integral closure of $J$ in $R$, i.e., $\bar{J}$ is the ideal of $R$ consisting of all elements $x \in R$ which satisfy an equation $x^{n}+r_{1} x^{n-1}+\cdots+r_{n}=0$, where $r_{i} \in J^{i}, i=1, \ldots, n$.

In his famous paper [11], D. Rees showed that a local ring $(R, \mathfrak{m})$ is analytically unramified if and only if the topology defined by $\overline{I^{n}}, n \geqslant 1$, is equivalent to the $I$-adic topology for an m-primary ideal $I$ of $R$. In [9], L. J. Ratliff, Jr., proved corresponding results in order to characterize reduced unmixed local rings. (Recall that a local ring $(R, \mathfrak{m})$ is called analytically unramified (resp. unmixed), if the $\mathfrak{m}$-adic completion, $R^{*}$, of $R$ is reduced (resp. all the prime ideals of $\operatorname{Ass}_{R^{*}} R^{*}$ have the same dimension). The main theorem of this paper gives a characterization of locally quasi-unmixed Noetherian rings, which is closely related to Ress' result [11]. Since such rings occur in many investigations in commutative algebra and algebraic geometry, it is desirable to know as many properties of such ring as possible. This characterization gives one such property, and that such rings

\footnotetext{
*Corresponding Author.

Email addresses: naghipour@ipm.ir, naghipour@tabrizu.ac.ir (R. Naghipour), adeleh_azari@yahoo.com (A. Azari), mahmoudi.simin@yahoo.com (S. Mollamahmoudi)

Received: 19.7.2017; Accepted: 12.8.2018
} 
have this property is a new result, and until now was not know to hold even in a regular local ring. More precisely we shall show that:

Theorem 1.1. Let $R$ denote a commutative Noetherian ring. Then the following conditions are equivalent:

(i) $R$ is locally quasi-unmixed.

(ii) For every ideal $I$ of the principal class in $R$, the topologies defined by $\overline{I^{n}}$ and $I^{\langle n\rangle}$, $n \geq 1$, are linearly equivalent.

(iii) For every ideal I of the principal class in $R$, the topologies defined by $\overline{I^{n}}$ and $I^{\langle n\rangle}$, $n \geq 1$, are equivalent.

This is closely related to Rees' result [11] for characterization quasi-unmixed local rings. Here $I^{\langle n\rangle}$ denotes the union $\left(\overline{I^{n}}:_{R} s\right)$, where $s$ varies in $R \backslash \bigcup\left\{\mathfrak{p} \in \operatorname{mAss}_{R} R / I\right\}$. See Theorem 2.5 for the proof of Theorem 1.1.

One of our tools for proving Theorem 1.1 is the following, which is a characterization of the equivalence between the topologies defined by the filtration $\overline{I^{n}}$ and $I^{\langle n\rangle}, n \geqslant 1$.

Proposition 1.2. Let I denote an ideal in a commutative Noetherian ring $R$. Then the topologies defined by $\overline{I^{n}}$ and $I^{\langle n\rangle}, n \geq 1$, are equivalent (resp. linearly equivalent) if and only if $\bar{Q}^{*}(I)$ (resp. $\left.\bar{A}^{*}(I)\right)$ is equal to $\mathrm{mAss}_{R} R / I$.

Pursuing this point of view further we prove some results about the behavior of the linearly equivalent topologies of ideals under various ring homomorphisms. In connection to this we derive the following consequence of Proposition 1.2.

Corollary 1.3. Let $R$ be a Noetherian ring and let $T$ be a finitely generated integral ring extension of $R$ such that every minimal prime of $T$ lies over a minimal prime of $R$. If the topologies $\overline{I^{n}}$ and $I^{\langle n\rangle}, n \geqslant 1$, are linearly equivalent, then the topologies defined by $\overline{(I T)^{n}}$ and $(I T)^{\langle n\rangle}, n \geqslant 1$, are also linearly equivalent; and the converse holds whenever $T$ is faithfully flat.

Throughout this paper, for any commutative Noetherian ring $R$ with nonzero identity, and for any ideal $I$ of $R$, we denote by $\operatorname{mAss}_{R} R / I$ the set of minimal prime ideals over $I$. If $(R, \mathfrak{m})$ is local, then $R^{*}$ denotes the completion of $R$ with respect to the $\mathfrak{m}$-adic topology. Then $R$ is said to be quasi-unmixed ring if for every $\mathfrak{p} \in \mathrm{mAss}_{R^{*}} R^{*}$, the condition $\operatorname{dim} R^{*} / \mathfrak{p}=\operatorname{dim} R$ is satisfied. More generally, if $R$ is not necessarily local, $R$ is a locally quasi-unmixed ring if for any $\mathfrak{p} \in \operatorname{Spec}(R), R_{\mathfrak{p}}$ is a local quasi-unmixed ring. For any ideal $I$ of $R$, we denote by $\mathscr{R}$ the graded Rees ring $R[u, I t]:=\bigoplus_{n \in \mathbb{Z}} I^{n} t^{n}$ of $R$ with respect to $I$, where $t$ is an indeterminate and $u=t^{-1}$. Also, the radical of $I$, denoted by $\operatorname{Rad}(I)$, is defined to be the set $\left\{x \in R \mid x^{n} \in I\right.$ for some $\left.n \in \mathbb{N}\right\}$. Finally, if $(R, \mathfrak{m})$ is local, then the analytic spread of $I$ is defined to be $\ell(I):=\operatorname{dim} \mathscr{R} /(\mathfrak{m}, u) \mathscr{R}$ (see [7]). For any unexplained notation and terminology we refer the reader to [1] or [5].

\section{Locally quasi-unmixed rings and comparison of topologies}

The purpose of this section is to establish a characterization of locally quasi-unmixed Noetherian rings, which is closely related to Ress' result [11]. The main goal is Theorem 2.5. The following lemmas are needed in the proof of that theorem.

Lemma 2.1. Let $I$ be an ideal of a Noetherian ring $R$. Then the following conditions are equivalent:

(i) $\bar{Q}^{*}(I)=\operatorname{mAss}_{R} R / I$.

(ii) The topologies defined by $\overline{I^{n}}$ and $I^{\langle n\rangle}, n \geq 1$, are equivalent. 
Proof. The assertion follows from the fact that $\operatorname{mAss}_{R} R / I \subseteq \bar{Q}^{*}(I)$ and $[3$, Theorem $1.5]$.

Lemma 2.2. Let $R$ be a Noetherian ring such that the topologies defined by $\overline{I^{n}}$ and $I^{\langle n\rangle}, n \geqslant 1$, are equivalent for all ideals $I$ of the principal class in $R$. Then for every prime ideal $\mathfrak{p}$ of $R$ and every ideal $J$ of the principal class in $R_{\mathfrak{p}}$, the topologies defined by $\overline{J^{n}}$ and $J^{\langle n\rangle}, n \geqslant 1$, are equivalent.

Proof. Let $\mathfrak{p} \in \operatorname{Spec}(R)$ and let $J$ be an ideal of the principal class in $R_{\mathfrak{p}}$. Then in view of [12, Lemma 5.1], there exists an ideal $I$ of $R$ of the principal class such that $J=I R_{\mathfrak{p}}$. Now, in view of Lemma 2.1, it is enough for us to show that $\bar{Q}^{*}(J)=\operatorname{mAss}_{R_{\mathfrak{p}}} R_{\mathfrak{p}} / J$. To do this, let $\mathfrak{q} R_{\mathfrak{p}} \in \bar{Q}^{*}(J)$. That is $\mathfrak{q} R_{\mathfrak{p}} \in \bar{Q}^{*}\left(I R_{\mathfrak{p}}\right)$. Then, by [3, Proposition 1.1], $\mathfrak{q} \in \bar{Q}^{*}(I)$, and so by Lemma $2.1, \mathfrak{q} \in \operatorname{mAss}_{R} R / I$. Therefore, $\mathfrak{q} R_{\mathfrak{p}} \in \operatorname{mAss}_{R_{\mathfrak{p}}} R_{\mathfrak{p}} / I R_{\mathfrak{p}}$, as required.

The next Lemma was proved by McAdam and Ratliff in [4].

Lemma 2.3. Let $I$ be an ideal of a locally quasi-unmixed Noetherian ring $R$ such that $\ell\left(I R_{\mathfrak{p}}\right)=\operatorname{height}\left(I R_{\mathfrak{p}}\right)$ for all $\mathfrak{p} \in \bar{A}^{*}(I)$. Then $\bar{A}^{*}(I)=\operatorname{mAss}_{R} R / I$.

Proof. See [4, Lemma 5.4].

Lemma 2.4. Let I denote an ideal in a Noetherian ring $R$. Then the following conditions are equivalent:

(i) $\bar{A}^{*}(I)=\operatorname{mAss}_{R} R / I$.

(ii) The topologies defined by $\overline{I^{n}}$ and $I^{\langle n\rangle}, n \geq 1$, are linearly equivalent.

Proof. The result follows from $\operatorname{mAss}_{R} R / I \subseteq \bar{A}^{*}(I)$ and [3, Corollary 1.6].

We are now ready to state and prove the main theorem of this section which is a characterization of locally quasi-unmixed Noetherian rings in terms of the equivalence (resp. linearly equivalence) between the topologies induced by $\overline{I^{n}}$ and $I^{\langle n\rangle}, n \geq 1$, for the principal class ideals $I$ of $R$. Recall that an ideal $I$ of $R$ is called of the principal class if $I$ is generated by height $I$ elements.

Theorem 2.5. Let $R$ denote a commutative Noetherian ring. Then the following conditions are equivalent:

(i) $R$ is locally quasi-unmixed.

(ii) For every ideal I of the principal class in $R$, the topologies defined by $\overline{I^{n}}$ and $I^{\langle n\rangle}$, $n \geq 1$, are linearly equivalent.

(iii) For every ideal I of the principal class in $R$, the topologies defined by $\overline{I^{n}}$ and $I^{\langle n\rangle}$, $n \geq 1$, are equivalent.

Proof. First we show (i) $\Longrightarrow$ (ii). If $R$ is locally quasi-unmixed, then in view of Lemmas 2.3 and 2.4 , it is enough for us to show that, for all $\mathfrak{p} \in \bar{A}^{*}(I), \ell\left(I R_{\mathfrak{p}}\right)=\operatorname{height}\left(I R_{\mathfrak{p}}\right)$ for every ideal $I$ of the principal class. To this end, in view of [2, Proposition 4.1], height $(\mathfrak{p})=\ell\left(I R_{\mathfrak{p}}\right)$. Now, since at least $\ell(\mathfrak{a})$ elements are needed to generate $\mathfrak{a}$, for any ideal $\mathfrak{a}$ in a commutative Noetherian ring $A$, and as $I R_{\mathfrak{p}}$ is an ideal of the principal class in $R_{\mathfrak{p}}$, it follows that $\ell\left(I R_{\mathfrak{p}}\right) \leq$ height $\left(I R_{\mathfrak{p}}\right)$. Furthermore, since $I \subseteq \mathfrak{p}$, it yields that

$$
\operatorname{height}\left(I R_{\mathfrak{p}}\right) \leq \operatorname{height}\left(\mathfrak{p} R_{\mathfrak{p}}\right)=\operatorname{height}(\mathfrak{p}),
$$

and so

$$
\ell\left(I R_{\mathfrak{p}}\right) \leq \operatorname{height}\left(I R_{\mathfrak{p}}\right) \leq \operatorname{height}(\mathfrak{p})=\ell\left(I R_{\mathfrak{p}}\right) .
$$

Hence $\ell\left(I R_{\mathfrak{p}}\right)=\operatorname{height}\left(I R_{\mathfrak{p}}\right)$, as required. 
Now, because of the implication (ii) $\Longrightarrow$ (iii) is trivially true, so in order to complete the proof we have to show that (iii) $\Longrightarrow$ (i). Let $\mathfrak{p} \in \operatorname{Spec}(R)$. We need to show that $R_{\mathfrak{p}}$ is a quasi-unmixed ring. To do this, in view of Lemma 2.2 and [8, Remark 2.9], without loss of generality we may assume that $(R, \mathfrak{m})$ is a local ring. Now, for proving the quasi-unmixedness of $R$, there are two cases to consider.

Case 1. Suppose that $\mathfrak{m} R^{*} \in \operatorname{mAss}_{R^{*}} R^{*}$. Then height $\left(\mathfrak{m} R^{*}\right)=0$, and so $\operatorname{dim} R^{*}=0$. Hence, $R$ is a quasi-unmixed ring, as required.

Case 2. Now, suppose that $\mathfrak{m} R^{*} \notin \mathrm{mAss}_{R^{*}} R^{*}$, and let $\mathfrak{q} \in \operatorname{mAss}_{R^{*}} R^{*}$. We need to show that $\operatorname{dim} R^{*} / \mathfrak{q}=\operatorname{dim} R$. To this end, as $\mathfrak{m} R^{*} \notin m \operatorname{mss}_{R^{*}} R^{*}$, we have $\operatorname{dim} R^{*} / \mathfrak{q}:=n$, where $n>0$. Therefore in view of [6, Proposition 3.5], there exists an ideal $\mathfrak{a}$ of $R$ of the principal class of height $n$ and $\operatorname{Rad}\left(\mathfrak{a} R^{*}+\mathfrak{q}\right)=\mathfrak{m} R^{*}$. Whence, $\mathfrak{m} \in \bar{Q}^{*}(\mathfrak{a})$. Moreover, as $\mathfrak{a}$ is the principal class, it follows from assumption (iii) and Lemma 2.1 that $\mathfrak{m} \in \operatorname{mAss}_{R} R / \mathfrak{a}$. Consequently, height $(\mathfrak{m})=n$, and $\operatorname{so} \operatorname{dim} R^{*} / \mathfrak{q}=\operatorname{dim} R$, as required.

The following corollary gives us a characterization of locally quasi-unmixed Noetherian rings in terms of quintasymptotic and presistent prime ideals of $I$.

Corollary 2.6. Let $R$ be a commutative Noetherian ring. Then the following conditions are equivalent:

(i) $R$ is locally quasi-unmixed.

(ii) $\bar{A}^{*}(I)=\operatorname{mAss}_{R} R / I$, for every ideal $I$ of the principal class of $R$.

(iii) $\bar{Q}^{*}(I)=\operatorname{mAss}_{R} R / I$, for every ideal $I$ of the principal class of $R$.

Proof. The assertion follows from Theorem 2.5, Lemma 2.1 and [3, Lemma 2.1].

As the final result of this section, we construct an example to show that the Theorem 2.5 is not true, if $I$ is not ideal of the principal class. The following lemma is needed in the proof of the Example 2.8.

Lemma 2.7. Let $R$ be a Noetherian ring such that $\operatorname{dim} R>0$. Let $I \subseteq \mathfrak{p}$ be ideals of $R$ with $\mathfrak{p} \in \operatorname{Spec}(R)$. Then the following conditions are equivalent:

(i) $\mathfrak{p} \in \bar{A}^{*}(I)$.

(ii) $\mathfrak{p} \in \bar{A}^{*}(x I)$ for any element $x$ not contained in any minimal prime of $R$.

Proof. See [2, Proposition 3.26].

Example 2.8. Let $k$ be a field and let $R=k[x, y]_{(x, y)}$. Set $\mathfrak{m}=(x, y) R$ and $I=x \mathfrak{m}$. Then $\mathfrak{m} \in \bar{A}^{*}(I)$ and $\mathfrak{m} \notin \bar{Q}^{*}(I)$.

Proof. Since $x$ is not contained in any minimal prime of $R$ and $\mathfrak{m} \in \bar{A}^{*}(\mathfrak{m})$, it follows from Lemma 2.7 that $\mathfrak{m} \in \bar{A}^{*}(I)$. Now, we need to show that $\mathfrak{m} \notin \bar{Q}^{*}(I)$. Suppose, the contrary, that $\mathfrak{m} \in \bar{Q}^{*}(I)$. Then, there exists $z \in \mathrm{mAss}_{R^{*}} R^{*}$ such that $\operatorname{Rad}\left(I R^{*}+z\right)=\mathfrak{m} R^{*}$. Since $I=x \mathfrak{m}$, it yields that $\operatorname{Rad}\left(x R^{*}+z\right)=\mathfrak{m} R^{*}$. Hence, $\mathfrak{m} R^{*} / z$ is minimal over $x\left(R^{*} / z\right)$, and so in view of Krull's Principal Ideal Theorem, height $\left(\mathfrak{m} R^{*} / z\right) \leq 1$. On the other hand, as $R^{*}$ is a Cohen-Macaulay ring, it follows that

$$
\operatorname{height}\left(\mathfrak{m} R^{*} / z\right)=\operatorname{height}\left(\mathfrak{m} R^{*}\right)-\operatorname{height}(z),
$$

and so height $\left(\mathfrak{m} R^{*} / z\right)=2$, which provides a contradiction. 


\section{Linearly equivalent topologies}

Our aim of this section is to obtain some results about the behavior of the linearly equivalent topologies of ideals under various ring homomorphisms.

Proposition 3.1. Let $R$ be a Noetherian ring and let $I$ be an ideal of $R$ such that the topologies defined by $\overline{I^{n}}$ and $I^{\langle n\rangle}, n \geq 1$, are linearly equivalent. Let $T$ be a finitely generated integral ring extension of $R$ such that every minimal prime of $T$ lies over a minimal prime of $R$. Then the topologies defined by $\overline{(I T)^{n}}$ and $(I T)^{\langle n\rangle}, n \geq 1$, are linearly equivalent.

Proof. In view of Lemma 2.4, it is enough to show that $\bar{A}^{*}(I T)=\mathrm{mAss}_{T} T / I T$. To this end, let $\mathfrak{p} \in \bar{A}^{*}(I T)$ and we show that $\mathfrak{p} \in \operatorname{mAss}_{T} T / I T$. Suppose the contrary that $\mathfrak{p} \notin \operatorname{mAss}_{T} T / I T$. Then, there exists $\mathfrak{q} \in \operatorname{mAss}_{T} T / I T$ such that $\mathfrak{q} \varsubsetneqq \mathfrak{p}$. Now as $\mathfrak{p}, \mathfrak{q} \in \bar{A}^{*}(I T)$, ( note that $\operatorname{mAss}_{T} T / I T \subseteq \bar{A}^{*}(I T)$ ), it follows from [10, Theorem 3.3] that $\mathfrak{p} \cap R$ and $\mathfrak{q} \cap R$ are contained in $\bar{A}^{*}(I)$. Hence, in view of Lemma 2.4, $\mathfrak{p} \cap R$ and $\mathfrak{q} \cap R$ are contained in $\operatorname{mAss}_{R} R / I$, and so $\mathfrak{p} \cap R=\mathfrak{q} \cap R$. Therefore, by [1, Theorem 9.3], $\mathfrak{p}=\mathfrak{q}$ which is a contradiction.

Proposition 3.2. Let $R$ be a Noetherian ring and let $I$ be an ideal of $R$. Let $T$ be a faithfully flat ring extension of $R$ such that the topologies defined by $\overline{(I T)^{n}}$ and $(I T)^{\langle n\rangle}, n \geq$ 1 , are linearly equivalent. Then the topologies defined by $\overline{I^{n}}$ and $I^{\langle n\rangle}, n \geq 1$, are linearly equivalent.

Proof. In view of Lemma 2.4 , it is enough to show that $\bar{A}^{*}(I)=\mathrm{mAss}_{R} R / I$. To do this, let $\mathfrak{p} \in \bar{A}^{*}(I)$. Then in view of $\left[9\right.$, Corollary 6.9], there exists $\mathfrak{p}^{*} \in \bar{A}^{*}(I T)$ such that $\mathfrak{p}^{*} \cap R=\mathfrak{p}$. Now by virtue of Lemma $2.4, \mathfrak{p}^{*} \in \mathrm{mAss}_{T} T / I T$. Hence, by the Going Down property between $T$ and $R$ (cf. [1, Theorem 9.5]), we see that $\mathfrak{p} \in \operatorname{mAss}_{R} R / I$, as required.

Theorem 3.3. Let $R$ be a Noetherian ring and let $I$ be an ideal of $R$. Then the topologies defined by $\overline{I^{n}}$ and $I^{\langle n\rangle}, n \geq 1$, are linearly equivalent if only if the topologies defined by $\overline{(I R[x])^{n}}$ and $(\operatorname{IR}[x])^{\langle n\rangle}, n \geq 1$, are linearly equivalent.

Proof. Since $R[x]$ is a faithfully flat ring extension of $R$, the sufficiency follows from Proposition 3.2. For necessity, in view of Lemma 2.4, it is enough to show that

$$
\bar{A}^{*}(I R[x])=\operatorname{mAss}_{R[x]} R[x] / I R[x] .
$$

To this end, let $\mathfrak{p} R[x] \in \bar{A}^{*}(\operatorname{IR}[x])$, note that by [2, Proposition 3.21],

$$
\bar{A}^{*}(I R[x])=\left\{\mathfrak{p} R[x] \mid \mathfrak{p} \in \bar{A}^{*}(I)\right\} .
$$

Then $\mathfrak{p} \in \bar{A}^{*}(I)$, and so by Lemma $2.4, \mathfrak{p} \in \operatorname{mAss}_{R} R / I$. Now, it easy to see that $\mathfrak{p} R[x] \in \operatorname{mAss}_{R[x]} R[x] / I R[x]$, as required.

Proposition 3.4. Let $R$ be a Noetherian ring and let $I$ be an ideal of $R$ such that the topologies defined by $\overline{I^{n}}$ and $I^{\langle n\rangle}, n \geq 1$, are linearly equivalent. Then for any $z \in$ $\operatorname{mAss}_{R} R / I$, the topologies defined by $\overline{(I+z / z)^{n}}$ and $(I+z / z)^{\langle n\rangle}, n \geq 1$, are linearly equivalent.

Proof. In view of Lemma 2.4, it suffices to show that

$$
\bar{A}^{*}(I+z / z)=\operatorname{mAss}_{R / z}((R / z) /(I+z / z)) .
$$


To do this, let $\mathfrak{p} / z \in \bar{A}^{*}(I+z / z)$. Then in view of [9, Corollary 6.3], $\mathfrak{p} \in \bar{A}^{*}(I)$. Hence, by Lemma $2.4, \mathfrak{p} \in \operatorname{mAss}_{R} R / I$. Now, it easy to see that

$$
\mathfrak{p} / z \in \operatorname{mAss}_{R / z}((R / z) /(I+z / z))
$$

as required.

Acknowledgment. The authors are deeply grateful to the referee for careful reading and many useful suggestions. We also would like to thank Professor Hossein Zakeri for reading of the first draft and valuable discussions.

\section{References}

[1] H. Matsumura, Commutative Ring Theory, Cambridge University Press, 1986.

[2] S. McAdam, Asymptotic Prime Divisors, Lecture Notes in Math. 1023, SpringerVerlag, New York, 1983.

[3] S. McAdam, Quintasymptotic primes and four results of Schenzel, J. Pure Appl. Algebra 47, 283-298, 1987.

[4] S. McAdam and L. J. Ratliff Jr., On the asymptotic cograde of an ideal, J. Algebra 87, 36-52, 1984.

[5] M. Nagata, Local Rings, Interscience, New York, 1961.

[6] R. Naghipour, Locally unmixed modules and ideal topologes, J. Algebra 236, 768-777, 2001.

[7] D.G. Northcott and D. Rees, Reductions of ideals in local rings, Proc. Cambridge Philos. Soc. 50, 145-158, 1954.

[8] L.J. Ratliff Jr., Asymptotic sequences, J. Algebra 85, 337-360, 1983.

[9] L.J. Ratliff Jr., On asymptotic prime divisors, Pacific J. Math. 111, 395-413, 1984.

[10] L.J. Ratliff Jr., Asymptotic prime divisors and integral extension rings, J. Algebra 95, 409-431, 1985.

[11] D. Rees, A note on analytically unramified local rings, J. London Math. Soc. 36, 24-28, 1961.

[12] J.K. Verma, On ideals whose adic and symbolic topologies are linearly equivalent, J. Pure Appl. Algebra 47, 205-212, 1987. 